TAIWANESE JOURNAL OF MATHEMATICS

Vol. 14, No. 4, pp. 1309-1324, August 2010

This paper is available online at http://www.tjm.nsysu.edu.tw/

\title{
STABILITY OF A MIXED ADDITIVE AND QUADRATIC FUNCTIONAL EQUATION IN NON-ARCHIMEDEAN BANACH MODULES
}

\author{
G. Zamani Eskandani, Hamid Vaezi and Y. N. Dehghan
}

\begin{abstract}
In this paper we establish the general solution of mixed additive and quadratic functional equation

$$
f(x+2 y)+f(x-2 y)+8 f(y)=2 f(x)+4 f(2 y)
$$

and investigate the generalized Hyers-Ulam-Rassias stability of this equation in non-Archimedean Banach modules over a unital Banach algebra.
\end{abstract}

\section{Introduction AND PReliminaries}

In 1940, S.M. Ulam [25] asked the first question on the stability problem. In 1941, D. H. Hyers [10] solved the problem of Ulam. This result was generalized by Aoki [4] for additive mappings and by Rassias [23] for linear mappings by considering an unbounded Cauchy difference. The paper of Rassias [23] has provided a lot of influence in the development of what we now call Hyers-Ulam-Rassias stability of functional equations. Since then, several stability problems for various functional equations have been investigated by numerous mathematicians; cf. e.g. $[6,11]$ and the bibliography quoted there. The functional equation

$$
f(x+y)+f(x-y)=2 f(x)+2 f(y)
$$

is related to a symmetric bi-additive function [15]. It is natural that such equation is called a quadratic functional equation. In particular, every solution of the quadratic equation (1.1) is said to be a quadratic function. It is well known that a function $f$

Received March 14, 2008, accepted August 25, 2008.

Communicated by Sen-Yen Shaw.

2000 Mathematics Subject Classification: Primary 39B72, 46B03, 47Jxx.

Key words and phrases: Hyers-Ulam-Rassias stability, Additive mapping, Quadratic mapping, Banach modules, Non-Archimedean space. 
between real vector spaces is quadratic if and only if there exists a unique symmetric biadditive function $B$ such that $f(x)=B(x, x)$ for all $x$ (see [15]). The biadditive function $B$ is given by

$$
B(x, y)=\frac{1}{4}(f(x+y)-f(x-y)) .
$$

A Hyers-Ulam stability problem for the quadratic functional equation (1.1) was proved by Skof for functions $f: E_{1} \rightarrow E_{2}$, where $E_{1}$ is a normed space and $E_{2}$ a Banach space (see [24]). Cholewa [5] noticed that the theorem of Skof is still true if the relevant domain $E_{1}$ is replaced by an Abelian group. In [7], Czerwik proved the Hyers-Ulam-Rassias stability of the quadratic functional equation (1.1). Grabiec in [9] has generalized the above mentioned results. Jun and Lee [13] proved the HyersUlam-Rassias stability of the pexiderized quadratic equation (1.1). Moslehian [18] and Mirzavazir [18] have investigated the orthogonal stability of the pexiderized quadratic equation (1.1).

By a non-Archimedean field we mean a field $K$ equipped with a function (valuation) $|$.$| from K$ into $[0, \infty)$ such that $|r|=0$ if and only if $r=0,|r s|=|r||s|$, and $|r+s| \leq \max \{|r|,|s|\}$ for all $r, s \in K$. Clearly $|1|=|-1|=1$ and $|n| \leq 1$ for all $n \in N$.

Let $X$ be a vector space over a scalar field $K$ with a non-Archimedean nontrivial valuation $\mid$. |. A function $\|\|:. X \longrightarrow \mathbb{R}$ is a non-Archimedean norm (valuation) if it satisfies the following conditions:

(i) $\|x\|=0$ if and only if $x=0$;

(ii) $\|r x\|=|r|\|x\|(r \in K, x \in X)$;

(iii) the strong triangle inequality; namely,

$$
\|x+y\| \leq \max \{\|x\|,\|y\|\} \quad(x, y \in X)
$$

Then $(X,\|\|)$ is called a non-Archimedean space. For nontrivial example of non-Archimedean space we refer to [17]. Due to the fact that

$$
\left\|x_{n}-x_{m}\right\| \leq \max \left\{\left\|x_{j+1}-x_{j}\right\|: m \leq j \leq n-1\right\} \quad(n>m)
$$

a sequence $\left\{x_{n}\right\}$ is Cauchy if and only if $\left\{x_{n+1}-x_{n}\right\}$ converges to zero in a nonArchimedean space. By a complete non-Archimedean space we mean one in which every Cauchy sequence is convergent.

In [17] Moslehian and Rassias have solved the stability problem for Cauchy and quadratic functional equations in non-Archimedean normed spaces. Stability of two types of cubic functional equations in non-Archimedean spaces have been investigated in [19]. In this context we refer to Arriola and Beyer [3] and Kaiser [14]. 
G. Z. Eskandani [8] has investigated the Hyers-Ulam-Rassias stability of the following functional equation

$$
\sum_{i=1}^{m} f\left(m x_{i}+\sum_{j=1, j \neq i}^{m} x_{j}\right)+f\left(\sum_{i=1}^{m} x_{i}\right)=2 f\left(\sum_{i=1}^{m} m x_{i}\right)
$$

$(m \in \mathbb{N}, m \geq 2)$ in quasi-Banach spaces.(See also [21, 22])

In this paper, using extensively the ideas and termonolgy of [17], we deal with the following functional equation deriving from additive and quadratic functions:

$$
f(x+2 y)+f(x-2 y)+8 f(y)=2 f(x)+4 f(2 y)
$$

It is easy to see that the function $f(x)=a x^{2}+b x$ is a solution of the functional equation (1.3) . The main purpose of this paper is to establish the general solution of Eq. (1.3) and investigate the Hyers-Ulam-Rassias stability for Eq. (1.3) in non-Archimedean Banach modules over a unital Banach algebra.

\section{Solutions OF EQ. (1.3)}

Throughout this section, $X$ and $Y$ will be real vector spaces. Before proceeding the proof of Theorem (2.3) which is the main result in this section, we shall need the following two lemmas.

Lemma 2.1. If an odd function $f: X \rightarrow Y$ satisfies (1.3) for all $x, y \in X$, then the mapping $f: X \rightarrow Y$ is additive .

Proof. Let an odd function $f: X \longrightarrow Y$ satisfy the functional equation (1.3) for all $x, y \in X$. Setting $x=0$ in (1.3), we get

$$
f(2 y)=2 f(y)
$$

for all $y \in X$. Therefore we have

$$
f(x+2 y)+f(x-2 y)=2 f(x)
$$

for all $x, y \in X$. Replace $x$ by $2 x$ in (2.2), we get

$$
f(x+y)+f(x-y)=2 f(x)
$$

for all $x, y \in X$. Replace $x$ and $y$ by $y$ and $x$ in (2.3), respectively, we get

$$
f(x+y)-f(x-y)=2 f(y)
$$

for all $x, y \in X$. Adding (2.3) to (2.4), we get

$$
f(x+y)=f(x)+f(y)
$$

for all $x, y \in X$. Therefore the function $f: X \rightarrow Y$ is additive. 
Lemma 2.2. If an even function $f: X \rightarrow Y$ satisfies (1.3) for all $x, y \in X$, then the mapping $f: X \rightarrow Y$ is quadratic.

Proof. Let an even function $f: X \longrightarrow Y$ satisfy the functional equation (1.3) for all $x, y \in X$. Putting $x=y=0$ in (1.3), we get $f(0)=0$. Setting $x=0$ in (1.3), we get

$$
f(2 y)=4 f(y)
$$

for all $y \in X$. Therefore we have

$$
f(x+2 y)+f(x-2 y)=2 f(x)+8 f(y)
$$

for all $x, y \in X$. Replace $x$ by $2 x$ in (2.6), we get

$$
f(x+y)+f(x-y)=2 f(x)+2 f(y)
$$

for all $x, y \in X$. Therefore the function $f: X \rightarrow X$ is quadratic.

Theorem 2.3. A function $f: X \rightarrow Y$ satisfies (1.3) for all $x, y \in X$ if and only if there exists a symmetric bi-additive function $B: X \times X \rightarrow Y$ and an additive function $A: X \rightarrow Y$ such that $f(x)=B(x, x)+A(x)$ for all $x \in X$.

Proof. If there exists a symmetric bi-additive function $B: X \times X \rightarrow Y$ and an additive function $A: X \rightarrow Y$ such that $f(x)=B(x, x)+A(x)$ for all $x \in X$, Then by a simple computation one can show that the functions $B$ and $A$ satisfy the functional equation (1.3). So the function $f$ satisfies the equation (1.3).

Conversely, we decompose $f$ into the even part and odd part by putting

$$
f_{e}(x)=\frac{f(x)+f(-x)}{2} \quad \text { and } \quad f_{o}(x)=\frac{f(x)-f(-x)}{2}
$$

for all $x \in X$. It is clear that $f(x)=f_{e}(x)+f_{o}(x)$ for all $x \in X$. It is easy to show that the functions $f_{e}$ and $f_{o}$ satisfy (1.3). Hence by Lemma 2.1 and Lemma 2.2 we achieve that the functions $f_{e}$ and $f_{o}$ are quadratic and additive, respectively. Therefore there exists a symmetric bi-additive function $B: X \times X \rightarrow Y$ such that $f_{e}(x)=B(x, x)$ for all $x \in X$ (see [1]). So

$$
f(x)=B(x, x)+A(x)
$$

for all $x \in X$, where $A(x)=f_{o}(x)$ for all $x \in X$. 


\section{Hyers-Ulam-Rassias Stability of EQ. (1.3)}

Throughout this section, let $B$ be a unital Banach algebra with norm $|$.$| and$ let ${ }_{B} X$ be left Banach $B$-module with norm $\|$. $\|$ and ${ }_{B} Y$ be non-Archimedean left Banach $B$-module with norm $\|$.$\| . In this section, we prove the stability of$ Eq. (1.3) in the spirit of Hyers, Ulam and Rassias. For convenience, we use the following abbreviations for a given function $f:{ }_{B} X \rightarrow{ }_{B} Y$ :

$$
\begin{aligned}
D_{a} f(x, y):= & f(a x+2 a y)+f(a x-2 a y)+8 f(a y)-2 a f(x)-4 a f(2 y) \\
\Delta_{a} f(x, y):= & f(a x+2 a y)+f(a x-2 a y)+8 f(a y)-2 a^{2} f(x)-4 a^{2} f(2 y) \\
M_{a} f(x, y):= & f(a x+2 a y)+f(a x-2 a y)+8 f(a y) \\
& -\left(a^{2}+a\right) f(x)-\left(a^{2}-a\right) f(-x)-2\left(a^{2}+a\right) f(2 y)-2\left(a^{2}-a\right) f(-2 y)
\end{aligned}
$$

for all $x, y \in{ }_{B} X$ and $a \in B$.

Theorem 3.1. Let $\varphi:{ }_{B} X \times{ }_{B} X \rightarrow[0, \infty)$ be a mapping such that

$$
\lim _{n \rightarrow \infty} \frac{1}{|2|^{n}} \varphi\left(2^{n} x, 2^{n} y\right)=0
$$

for all $x, y \in{ }_{B} X$ and

$$
\tilde{\varphi}(x):=\sup \left\{\frac{\varphi\left(0,2^{j} x\right)}{|2|^{j}}: j \in N \cup\{0\}\right\}
$$

exists for all $x \in{ }_{B} X$. Suppose that an odd mapping $f:{ }_{B} X \rightarrow_{B} Y$ satisfies the inequality

$$
\left\|D_{a} f(x, y)\right\| \leq \varphi(x, y)
$$

for all $x, y \in{ }_{B} X$ and $a \in B$. Then there exists a unique additive mapping $A:{ }_{B} X \rightarrow{ }_{B} Y$ satisfying $A(a x)=a A(x)$ for all $x \in{ }_{B} X$ and $a \in B$ such that

$$
\|f(x)-A(x)\| \leq \frac{1}{|8|} \tilde{\varphi}(x)
$$

for all $x \in{ }_{B} X$.

Proof. Putting $x=0$ and $a=1$ in (3.3), we get

$$
\|f(2 y)-2 f(y)\| \leq \frac{1}{|4|} \varphi(0, y)
$$


for all $y \in{ }_{B} X$. Replacing $y$ by $2^{n} x$ in (3.5) and dividing both sides of (3.5) by $|2|^{n+1}$, we get

$$
\left\|\frac{1}{2^{n+1}} f\left(2^{n+1} x\right)-\frac{1}{2^{n}} f\left(2^{n} x\right)\right\| \leq \frac{1}{|2|^{n+3}} \varphi\left(0,2^{n} x\right)
$$

for all $x \in{ }_{B} X$ and all non-negative integers $n$. Therefore, we conclude from (3.1) and (3.6) that the sequence $\left\{\frac{1}{2^{n}} f\left(2^{n} x\right)\right\}$ is a Cauchy sequence in ${ }_{B} Y$ for all $x \in{ }_{B} X$. Since ${ }_{B} Y$ is complete the sequence $\left\{\frac{1}{2^{n}} f\left(2^{n} x\right)\right\}$ converges in ${ }_{B} Y$ for all $x \in{ }_{B} X$. So one can define the mapping $A:{ }_{B} X \rightarrow{ }_{B} Y$ by

$$
A(x):=\lim _{n \rightarrow \infty} \frac{1}{2^{n}} f\left(2^{n} x\right)
$$

for all $x \in{ }_{B} X$. Using induction one can show that

$$
\left\|\frac{f\left(2^{n} x\right)}{2^{n}}-f(x)\right\| \leq \frac{1}{|8|} \max \left\{\frac{\varphi\left(0,2^{j} x\right)}{|2|^{j}}: 0 \leq j<n\right\}
$$

for all $n \in N$ and all $x \in{ }_{B} X$. By taking $n$ to approach infinity in (3.8) and using (3.2) and (3.7) one obtains (3.4). Now, we show that $A$ is an additive mapping. It follows from (3.1), (3.3) and (3.7) that

$$
\left\|D_{1} A(x, y)\right\|=\lim _{n \rightarrow \infty} \frac{1}{|2|^{n}}\left\|D_{1} f\left(2^{n} x, 2^{n} y\right)\right\| \leq \lim _{n \rightarrow \infty} \frac{1}{|2|^{n}} \varphi\left(2^{n} x, 2^{n} y\right)=0
$$

for all $x, y \in{ }_{B} X$. Hence, the mapping $A$ satisfies (1.3), So by Lemma (2.1) the mapping $A$ is additive. Replacing $y$ by 0 in (3.3), we get

$$
\|f(a x)-a f(x)\| \leq \frac{1}{|2|} \varphi(x, 0)
$$

for all $x \in{ }_{B} X$ and $a \in B$. Replacing $x$ by $2^{n} x$ in (3.9) and dividing both sides of (3.9) by $|2|^{n}$, we get

$$
\left\|\frac{1}{2^{n}} f\left(2^{n} a x\right)-\frac{a}{2^{n}} f\left(2^{n} x\right)\right\| \leq \frac{1}{|2|^{n+1}} \varphi\left(2^{n} x, 0\right)
$$

for all $x \in{ }_{B} X, a \in B_{1}$ and for all non-negative integers $n$. Therefor

$$
\left\|\frac{1}{2^{n}} f\left(2^{n} a x\right)-\frac{a}{2^{n}} f\left(2^{n} x\right)\right\| \longrightarrow 0
$$

as $n \longrightarrow \infty$ for all $x \in{ }_{B} X, a \in B$. Hence

$$
A(a x)=\lim _{n \rightarrow \infty} \frac{1}{2^{n}} f\left(2^{n} a x\right)=\lim _{n \rightarrow \infty} \frac{a}{2^{n}} f\left(2^{n} x\right)=a A(x)
$$


for all $x \in{ }_{B} X$ and $a \in B$.

To prove the uniqueness of $A$, let $T:{ }_{B} X \rightarrow{ }_{B} Y$ be another additive mapping satisfying (3.4). Then

$$
\begin{aligned}
\|A(x)-T(x)\| & =\lim _{k \rightarrow \infty}|2|^{-k}\left\|A\left(2^{k} x\right)-T\left(2^{k} x\right)\right\| \\
& \leq \lim _{k \rightarrow \infty}|2|^{-k} \max \left\{\left\|A\left(2^{k} x\right)-f\left(2^{k} x\right)\right\|,\left\|T\left(2^{k} x\right)-f\left(2^{k} x\right)\right\|\right\} \\
& \leq \frac{1}{|8|} \lim _{k \rightarrow \infty} \lim _{n \rightarrow \infty} \max \left\{\frac{\varphi\left(0,2^{j} x\right)}{|2|^{j}}: k \leq j<n+k\right\} \\
& =\frac{1}{|8|} \lim _{k \rightarrow \infty} \sup \left\{\frac{\varphi\left(0,2^{j} x\right)}{|2|^{j}}: k \leq j<\infty\right\}=0
\end{aligned}
$$

for all $x \in{ }_{B} X$. So $A=T$.

Theorem 3.2. Let $\Phi:{ }_{B} X \times{ }_{B} X \rightarrow[0, \infty)$ be a mapping such that

$$
\lim _{n \rightarrow \infty}|2|^{n} \Phi\left(\frac{x}{2^{n}}, \frac{y}{2^{n}}\right)=0
$$

for all $x, y \in{ }_{B} X$ and

$$
\widetilde{\Phi}(x):=\sup \left\{|2|^{j} \Phi\left(0, \frac{x}{2^{j+1}}\right): j \in N \cup\{0\}\right\}
$$

exists for all $x \in{ }_{B} X$. Suppose that an odd mapping $f:{ }_{B} X \rightarrow_{B} Y$ satisfies the inequality

$$
\left\|D_{a} f(x, y)\right\| \leq \Phi(x, y)
$$

for all $x, y \in{ }_{B} X$ and $a \in B$. Then there exists a unique additive mapping $A:{ }_{B} X \rightarrow{ }_{B} Y$ satisfying $A(a x)=a A(x)$ for all $x \in{ }_{B} X$ and $a \in B$ such that

$$
\|f(x)-A(x)\| \leq \frac{1}{|4|} \tilde{\Phi}(x)
$$

for all $x \in{ }_{B} X$.

Proof. Similar to the proof of Theorem 3.1, we have

$$
\|f(2 y)-2 f(y)\| \leq \frac{1}{|4|} \Phi(0, y)
$$

for all $y \in{ }_{B} X$. Replacing $y$ by $\frac{x}{2^{n+1}}$ in (3.13) and multiplying both sides of (3.13) to $|2|^{n}$, we get

$$
\left\|2^{n+1} f\left(\frac{x}{2^{n+1}}\right)-2^{n} f\left(\frac{x}{2^{n}}\right)\right\| \leq|2|^{n-2} \Phi\left(0, \frac{x}{2^{n+1}}\right)
$$


for all $x \in{ }_{B} X$ and all non-negative integers $n$. Therefor, we conclude from (3.10) and (3.14) that the sequence $\left\{2^{n} f\left(x / 2^{n}\right)\right\}$ is a Cauchy sequence in ${ }_{B} Y$ for all $x \in{ }_{B} X$. Since ${ }_{B} Y$ is complete the sequence $\left\{2^{n} f\left(x / 2^{n}\right)\right\}$ converges in ${ }_{B} Y$ for all $x \in{ }_{B} X$. So one can define the mapping $A:{ }_{B} X \rightarrow{ }_{B} Y$ by

$$
A(x):=\lim _{n \rightarrow \infty} 2^{n} f\left(\frac{x}{2^{n}}\right)
$$

for all $x \in{ }_{B} X$. Using induction one can show that

$$
\left\|2^{n} f\left(\frac{x}{2^{n}}\right)-f(x)\right\| \leq \frac{1}{|4|} \max \left\{|2|^{j} \Phi\left(0, \frac{x}{2^{j+1}}\right): 0 \leq j<n\right\}
$$

for all $n \in N$ and all $x \in H$. By taking $n$ to approach infinity in (3.16) and using (3.11) and (3.15) one obtains (3.12). The rest of the proof is similar to the proof of Theorem 3.1.

Corollary 3.3. Let $\theta, r, s$ be positive real numbers such that $r, s \neq 1$ and $|2|<1$. Suppose that an odd mapping $f:{ }_{B} X \rightarrow_{B} Y$ satisfies the inequality

$$
\left\|D_{a} f(x, y)\right\| \leq \theta\left(\|x\|^{r}+\|y\|^{s}\right)
$$

for all $x, y \in{ }_{B} X$ and $a \in B$. Then there exists a unique additive mapping $A:{ }_{B} X \rightarrow{ }_{B} Y$ satisfying $A(a x)=a A(x)$ for all $x \in{ }_{B} X$ and $a \in B$ such that

$$
\|f(x)-A(x)\| \leq \begin{cases}\frac{\theta\|x\|^{s}}{|8|} & (r, s>1) \\ \frac{\theta\|x\|^{s}}{|2|^{s+2}} & (r, s<1)\end{cases}
$$

for all $x \in{ }_{B} X$.

Corollary 3.4. Let $\theta, r, s$ be positive real numbers such that $r+s \neq 1$ and $|2|<1$. Suppose that an odd function $f:{ }_{B} X \rightarrow{ }_{B} Y$ satisfies the inequality

$$
\left\|D_{a} f(x, y)\right\| \leq \theta\|x\|^{r}\|y\|^{s}
$$

for all $a \in B$ and $x, y \in{ }_{B} X$. Then $f:{ }_{B} X \rightarrow{ }_{B} Y$ is an additive mapping satisfying $f(a x)=a f(x)$ for all $x \in{ }_{B} X$ and $a \in B$.

Theorem 3.5. Let $\varphi:{ }_{B} X \times{ }_{B} X \rightarrow[0, \infty)$ be a mapping such that

$$
\lim _{n \rightarrow \infty} \frac{\varphi\left(2^{n} x, 2^{n} y\right)}{|4|^{n}}=0
$$


for all $x, y \in{ }_{B} X$ and

$$
\widetilde{\varphi}(x):=\sup \left\{\frac{\varphi\left(0,2^{j} x\right)}{|4|^{j}}: j \in N \cup\{0\}\right\}
$$

exists for all $x \in{ }_{B} X$. Suppose that an even mapping $f:{ }_{B} X \rightarrow{ }_{B} Y$ with $f(0)=0$ satisfies the inequality

$$
\left\|\Delta_{a} f(x, y)\right\| \leq \varphi(x, y)
$$

for all $x, y \in{ }_{B} X$ and $a \in B$, Then there exists a unique quadratic mapping $Q:{ }_{B} X \rightarrow{ }_{B} Y$ satisfying $Q(a x)=a^{2} Q(x)$ for all $x \in{ }_{B} X$ and $a \in B$ such that

$$
\|f(x)-Q(x)\| \leq \frac{1}{|8|} \widetilde{\varphi}(x)
$$

for all $x \in{ }_{B} X$.

Proof. Putting $x=0, a=1$ in (3.19), we get

$$
\|f(2 y)-4 f(y)\| \leq \frac{1}{|2|} \varphi(0, y)
$$

for all $y \in{ }_{B} X$. Replacing $y$ by $2^{n} x$ in (3.21) and dividing both sides of (3.21) by $|4|^{n+1}$, we get

$$
\left\|\frac{1}{4^{n+1}} f\left(2^{n+1} x\right)-\frac{1}{4^{n}} f\left(2^{n} x\right)\right\| \leq \frac{1}{|2|^{2 n+3}} \varphi\left(0,2^{n} x\right)
$$

for all $x \in{ }_{B} X$ and all non-negative integers $n$. Therefore, we conclude from (3.17) and (3.22) that the sequence $\left\{\frac{1}{4^{n}} f\left(2^{n} x\right)\right\}$ is a Cauchy sequence in ${ }_{B} Y$ for all $x \in{ }_{B} X$. Since ${ }_{B} Y$ is complete the sequence $\left\{\frac{1}{4^{n}} f\left(2^{n} x\right)\right\}$ converges for all $x \in{ }_{B} X$. So one can define the mapping $Q:{ }_{B} X \rightarrow{ }_{B} Y$ by:

$$
Q(x)=\lim _{n \rightarrow \infty} \frac{1}{4^{n}} f\left(2^{n} x\right)
$$

for all $x \in{ }_{B} X$. Using induction one can show that

$$
\left\|\frac{f\left(2^{n} x\right)}{4^{n}}-f(x)\right\| \leq \frac{1}{|8|} \max \left\{\frac{\varphi\left(0,2^{j} x\right)}{|4|^{j}}: 0 \leq j<n\right\}
$$

for all $n \in N$ and all $x \in{ }_{B} X$. By taking $n$ to approach infinity in (3.24) and using (3.18) and (3.23) one obtains (3.20). 
Now, we show that the mapping $Q$ is quadratic. It follows from (3.17), (3.19) and (3.23) that

$$
\begin{aligned}
\left\|\Delta_{1} Q(x, y)\right\| & =\lim _{n \rightarrow \infty} \frac{1}{|4|^{n}}\left\|\Delta_{1} f\left(2^{n} x, 2^{n} y\right)\right\| \\
& \leq \lim _{n \rightarrow \infty} \frac{1}{|4|^{n}} \varphi\left(2^{n} x, 2^{n} y\right)=0
\end{aligned}
$$

for all $x, y \in{ }_{B} X$, So the mapping $Q$ satisfies (1.3), hence by Lemma 2.2, the mapping $Q$ is quadratic. Replacing $y=0$ in (3.19), we have

$$
\left\|f(a x)-a^{2} f(x)\right\| \leq \frac{1}{|2|} \varphi(x, 0)
$$

for all $x \in{ }_{B} X$ and $a \in B$. Replacing $x$ by $2^{n} x$ in (3.25) and dividing both sides of $(3.25)$ by $|4|^{n}$, we get

$$
\left\|\frac{1}{4^{n}} f\left(2^{n} a x\right)-\frac{a^{2}}{4^{n}} f\left(2^{n} x\right)\right\| \leq \frac{1}{|2|^{2 n+1}} \varphi\left(2^{n} x, 0\right)
$$

for all $x \in{ }_{B} X, a \in B$ and for all non-negative integers $n$. Therefore

$$
\left\|\frac{1}{4^{n}} f\left(2^{n} a x\right)-\frac{a^{2}}{4^{n}} f\left(2^{n} x\right)\right\| \longrightarrow 0
$$

as $n \longrightarrow \infty$ for all $x \in{ }_{B} X, a \in B_{1}$. Hence

$$
Q(a x)=\lim _{n \rightarrow \infty} \frac{1}{4^{n}} f\left(2^{n} a x\right)=\lim _{n \rightarrow \infty} \frac{1}{4^{n}} a^{2} f\left(2^{n} x\right)=a^{2} Q(x)
$$

for all $x \in{ }_{B} X, a \in B$. To prove the uniqueness of $Q$, let $T:{ }_{B} X \rightarrow{ }_{B} X$ be another quadratic mapping satisfying (3.20). So it follows from (3.20) and (3.23) that

$$
\begin{aligned}
\|Q(x)-T(x)\| & =\lim _{k \rightarrow \infty}|4|^{-k}\left\|Q\left(2^{k} x\right)-T\left(2^{k} x\right)\right\| \\
& \leq \lim _{k \rightarrow \infty}|4|^{-k} \max \left\{\left\|Q\left(2^{k} x\right)-f\left(2^{k} x\right)\right\|,\left\|T\left(2^{k} x\right)-f\left(2^{k} x\right)\right\|\right\} \\
& \leq \frac{1}{|8|} \lim _{k \rightarrow \infty} \lim _{n \rightarrow \infty} \max \left\{\frac{\varphi\left(0,2^{j} x\right)}{|4|^{j}}: k \leq j<n+k\right\} \\
& \leq \frac{1}{|8|} \lim _{k \rightarrow \infty} \sup \left\{\frac{\varphi\left(0,2^{j} x\right)}{|4|^{j}}: k \leq j<\infty\right\}=0
\end{aligned}
$$

for all $x \in{ }_{B} X$. So $Q=T$. 
Theorem 3.6. Let $\Phi:{ }_{B} X \times{ }_{B} X \rightarrow[0, \infty)$ be a mapping such that

$$
\lim _{n \rightarrow \infty}|4|^{n} \Phi\left(\frac{x}{2^{n}}, \frac{y}{2^{n}}\right)=0
$$

for all $x, y \in{ }_{B} X$ and

$$
\widetilde{\Phi}(x):=\sup \left\{|4|^{j} \Phi\left(0, \frac{x}{2^{j+1}}\right): j \in N \cup\{0\}\right\}
$$

exists for all $x \in{ }_{B} X$. Suppose that an even mapping $f:{ }_{B} X \rightarrow{ }_{B} Y$ with $f(0)=0$ satisfies the inequality

$$
\left\|\Delta_{a} f(x, y)\right\| \leq \Phi(x, y)
$$

for all $x, y \in{ }_{B} X$ and $a \in B$. Then there exists a unique quadratic mapping $Q:{ }_{B} X \rightarrow{ }_{B} Y$ satisfying $Q(a x)=a^{2} Q(x)$ for all $x \in{ }_{B} X$ and $a \in B$ such that

$$
\|f(x)-Q(x)\| \leq \frac{1}{|2|} \widetilde{\Phi}(x)
$$

for all $x \in{ }_{B} X$.

Proof. Similar to the proof of Theorem 3.5, we have

$$
\|f(2 y)-4 f(y)\| \leq \frac{1}{|2|} \Phi(0, y)
$$

for all $y \in{ }_{B} X$. Replacing $y$ by $\frac{x}{2^{n+1}}$ in (3.29) and multiplying both sides of (3.29) to $|4|^{n}$, we get

$$
\left\|4^{n+1} f\left(\frac{x}{2^{n+1}}\right)-4^{n} f\left(\frac{x}{2^{n}}\right)\right\| \leq|2|^{2 n-1} \Phi\left(0, \frac{x}{2^{n+1}}\right)
$$

for all $x \in{ }_{B} X$ and all non-negative integers $n$. Therefore we conclude from (3.26) and (3.30) that the sequence $\left\{4^{n} f\left(x / 2^{n}\right)\right\}$ is a Cauchy sequence in ${ }_{B} Y$ for all $x \in{ }_{B} X$. Since ${ }_{B} Y$ is complete the sequence $\left\{4^{n} f\left(x / 2^{n}\right)\right\}$ converges in ${ }_{B} Y$ for all $x \in{ }_{B} X$. So one can define the mapping $Q:{ }_{B} X \rightarrow{ }_{B} Y$ by

$$
Q(x):=\lim _{n \rightarrow \infty} 4^{n} f\left(\frac{x}{2^{n}}\right)
$$

for all $x \in{ }_{B} X$. Using induction one can show that

$$
\left\|4^{n} f\left(\frac{x}{2^{n}}\right)-f(x)\right\| \leq \frac{1}{|2|} \max \left\{|4|^{j} \Phi\left(0, \frac{x}{2^{j+1}}\right): 0 \leq j<n\right\}
$$

for all $n \in N$ and all $x \in{ }_{B} X$. By taking $n$ to approach infinity in (3.32) and using (3.27) and (3.31) one obtains (3.28). The rest of the proof is similar to Theorem 3.5. 
Corollary 3.7. Let $\theta, r, s$ be positive real numbers such that $r, s \neq 2$ and $|2|<1$. Suppose that an even mapping $f:{ }_{B} X \rightarrow_{B} Y$ satisfies the inequality

$$
\left\|\Delta_{a} f(x, y)\right\| \leq \theta\left(\|x\|^{r}+\|y\|^{s}\right)
$$

for all $x, y \in{ }_{B} X$ and $a \in B$. Then there exists a unique quadratic mapping $Q:{ }_{B} X \rightarrow{ }_{B} Y$ satisfying $Q(a x)=a^{2} Q(x)$ for all $x \in{ }_{B} X$ and $a \in B$ such that

$$
\|f(x)-Q(x)\| \leq\left\{\begin{array}{cc}
\frac{\theta\|x\|^{s}}{|8|} & (r, s>2) \\
\frac{\theta\|x\|^{s}}{|2|^{s+1}} & (r, s<2)
\end{array}\right.
$$

for all $x \in{ }_{B} X$.

Corollary 3.8. Let $\theta, r, s$ be positive real numbers such that $r+s \neq 2$ and $|2|<1$. Suppose that an even mapping $f:{ }_{B} X \rightarrow{ }_{B} Y$ satisfies the inequality

$$
\left\|\Delta_{a} f(x, y)\right\| \leq \theta\|x\|^{r}\|y\|^{s}
$$

for all $x, y \in{ }_{B} X$ and $a \in B$. Then $f:{ }_{B} X \rightarrow{ }_{B} Y$ is a quadratic mapping satisfying $f(a x)=a^{2} f(x)$ for all $x \in{ }_{B} X$ and $a \in B$.

We now prove our main theorem in this section.

Theorem 3.9. Let $\varphi:{ }_{B} X \times{ }_{B} X \rightarrow[0, \infty)$ be a mapping satisfying (3.17) for all $x, y \in{ }_{B} X$, and let

$$
\widetilde{\psi}_{m}(x):=\sup \left\{\frac{\varphi\left(0,2^{j} x\right)}{|m|^{j}}+\frac{\varphi\left(0,-2^{j} x\right)}{|m|^{j}}: j \in N \cup\{0\}\right\}
$$

exists for all $x \in{ }_{B} X$ and $m \in\{2,4\}$. Suppose that a mapping $f:{ }_{B} X \rightarrow{ }_{B} Y$ with $f(0)=0$ satisfies the inequality

$$
\left\|M_{a} f(x, y)\right\| \leq \varphi(x, y)
$$

for all $x, y \in{ }_{B} X$ and $a \in B$. Then there exists a unique additive mapping $A:{ }_{B} X \rightarrow{ }_{B} Y$ and a unique quadratic mapping $Q:{ }_{B} X \rightarrow{ }_{B} Y$ satisfying $A(a x)=a A(x), Q(a x)=a^{2} Q(x)$ for all $x \in{ }_{B} X$ and $a \in B$ such that

$$
\|f(x)-A(x)-Q(x)\| \leq \frac{1}{|16|} \max \left\{\widetilde{\psi}_{2}(x), \widetilde{\psi}_{4}(x)\right\}
$$

for all $x \in{ }_{B} X$. 
Proof. Let $f_{e}(x)=\frac{f(x)+f(-x)}{2}$ and $f_{o}(x)=\frac{f(x)-f(-x)}{2}$, then $f_{e}(-x)=$ $f_{e}(x)$ and $f_{o}(-x)=-f_{o}(x)$ for all $x \in{ }_{B} X$. Let

$$
\psi(x, y):=\frac{1}{|2|}[\varphi(x, y)+\varphi(-x,-y)]
$$

for all $x, y \in{ }_{B} X$. By using (3.34) and (3.36), we have

$$
\begin{aligned}
\left\|\Delta_{a} f_{e}(x, y)\right\| & =\left\|M_{a} f_{e}(x, y)\right\| \leq \psi(x, y) \\
\left\|D_{a} f_{o}(x, y)\right\| & =\left\|M_{a} f_{o}(x, y)\right\| \leq \psi(x, y)
\end{aligned}
$$

for all $x, y \in{ }_{B} X$ and $a \in B$. Hence, the result follows by using Theorems 3.1 and 3.5 for $\psi$ instead of $\varphi$.

Theorem 3.10. Let $\Phi:{ }_{B} X \times{ }_{B} X \rightarrow[0, \infty)$ be a mapping satisfying (3.10) for all $x, y \in{ }_{B} X$, and let

$$
\widetilde{\Psi}_{m}(x):=\sup \left\{|m|^{j} \Phi\left(0, \frac{x}{2^{j+1}}\right)+|m|^{j} \Phi\left(0,-\frac{x}{2^{j+1}}\right): j \in N \cup\{0\}\right\}
$$

exists for all $x \in{ }_{B} X$. Suppose that a mapping $f:{ }_{B} X \rightarrow_{B} Y$ with $f(0)=0$ satisfies the inequality

$$
\left\|M_{a} f(x, y)\right\| \leq \Phi(x, y)
$$

for all $x, y \in{ }_{B} X$ and $a \in B$. Then there exists a unique additive mapping $A:{ }_{B} X \rightarrow{ }_{B} Y$ and a unique quadratic mapping $Q:{ }_{B} X \rightarrow{ }_{B} Y$ satisfying $A(a x)=a A(x), Q(a x)=a^{2} Q(x)$ for all $x \in{ }_{B} X$ and $a \in B$ such that

$$
\|f(x)-A(x)-Q(x)\| \leq \frac{1}{|8|} \max \left\{\widetilde{\Psi}_{2}(x), \widetilde{\Psi}_{4}(x)\right\}
$$

for all $x \in{ }_{B} X$.

Proof. Similar to Theorem 3.9, the results can be obtained by using Theorems 3.2 and 3.6 .

Corollary 3.11. Let $\theta, r, s$ be positive real numbers such that $(r, s<1)$ or $(r, s>2)$ and $|2|<1$. Suppose that a mapping $f:{ }_{B} X \rightarrow{ }_{B} Y$ satisfies the inequality

$$
\left\|M_{a} f(x, y)\right\| \leq \theta\left(\|x\|^{r}+\|y\|^{s}\right)
$$

for all $x, y \in{ }_{B} X$ and $a \in B$. Then there exists a unique additive mapping $A:{ }_{B} X \rightarrow{ }_{B} Y$ and a unique quadratic mapping $Q:{ }_{B} X \rightarrow{ }_{B} Y$ satisfying 
$A(a x)=a A(x), Q(a x)=a^{2} Q(x)$ for all $x \in{ }_{B} X$ and $a \in B$ such that

$$
\|f(x)-A(x)-Q(x)\| \leq \begin{cases}\frac{2 \theta\|x\|^{s}}{|16|} & (r, s>2) \\ \frac{2 \theta\|x\|^{s}}{|2|^{s+3}} & (r, s<1)\end{cases}
$$

for all $x \in{ }_{B} X$.

Corollary 3.12. Let $\theta, r, s$ be positive real numbers such that $(r+s<1)$ or $(r+s>2)$ and $|2|<1$. Suppose that a mapping $f:{ }_{B} X \rightarrow_{B} Y$ satisfies the inequality

$$
\left\|M_{a} f(x, y)\right\| \leq \theta\|x\|^{r}\|y\|^{s}
$$

for all $x, y \in{ }_{B} X$ and $a \in B$. Then there exists a unique additive mapping $A:{ }_{B} X \rightarrow{ }_{B} Y$ and a unique quadratic mapping $Q:{ }_{B} X \rightarrow{ }_{B} Y$ satisfying $A(a x)=a A(x), Q(a x)=a^{2} Q(x)$ for all $x \in{ }_{B} X$ and $a \in B$ such that

$$
f(x)=A(x)+Q(x)
$$

for all $x \in{ }_{B} X$.

\section{ACKNOWLEDGMENTS}

The authors are very thankful to the referee for a number of valuable suggestions. Also, they would like to thank Prof. M. S. Moslehian for invaluable comments regarding to the previous version of the manuscript.

\section{REFERENCES}

1. J. Aczél and J. Dhombres, Functional Equations in Several Variables, Cambridge University Press, 1989.

2. D. Amir, Characterizations of Inner Product Spaces, Birkhäuser, Basel, 1986.

3. L. M. Arriola and W. A. Beyer, Stability of the Cauchy functional equation over p-adic fields, Real Analysis Exchange, 31 (2005/2006), 125-132.

4. T. Aoki, On the stability of the linear transformation in Banach spaces, J. Math. Soc. Japan, 2 (1950), 64-66.

5. P. W. Cholewa, Remarks on the stability of functional equations, Aequationes Math., 27 (1984), 76-86.

6. S. Czerwik, Functional equations and inequalities in several variables, World Scientific, New Jersey-London-Singapore-Hong Kong, 2002. 
7. S. Czerwik, On the stability of the quadratic mapping in normed spaces, Abh. Math. Sem. Univ. Hamburg 62 (1992), 59-64.

8. G. Z. Eskandani, On the Hyers-Ulam-Rassias stability of an additive functional equation in quasi-Banach spaces, J. Math. Anal. Appl., 345 (2008), 405-409.

9. A. Grabiec, The generalized Hyers-Ulam stability of a class of functional equations, Publ. Math. Debrecen, 48 (1996), 217-235.

10. D. H. Hyers, On the stability of the linear functional equation, Proc. Natl. Acad. Sci., 27 (1941), 222-224.

11. D. H. Hyers, G. Isac and Th. M. Rassias, Stability of functional equations in several variables, Birkhäuser, Basel, 1998.

12. P. Jordan and J. Von Neumann, On inner products in linear metric spaces, Ann. of Math., 36 (1935), 719-723.

13. K. Jun and Y. Lee, On the Hyers-Ulam-Rassias stability of a Pexiderized quadratic inequality, Math. Inequal. Appl., 4 (2001), 93-118.

14. Z. Kaiser, On stability of the Cauchy equation in normed spaces over fields with valuation, Publ. Math. Debrecen, 64(1-2) (2004), 189-200.

15. Pl. Kannappan, Quadratic functional equation and inner product spaces, Results Math., 27 (1995), 368-372.

16. M. Mirzavaziri and M. S. Moslehian, A fixed point approach to stability of a quadratic equation, Bull. Braz. Math. Soc., 37(3) (2006), 361-376.

17. M. S. Moslehian and Th. M. Rassias, Stability of functional equation in nonArchimedean spaces, Applicable Analysis and Discrete Mathematics, 1 (2007), 325334.

18. M. S. Moslehian, On the orthogonal stability of the Pexiderized quadratic equation, J. Differ. Equations. Appl., 11(11) (2005), 999-1004.

19. M. S. Moslehian and Gh. Sadegi, Stability of two types of cubic functional equations in non-Archimedean spaces, Real Anal. Exchange, 32(2) (2008), 375-383.

20. F. Moradlou, H. Vaezi and C. Park, Fixed Points and Stability of an Additive Functional Equation of n-Apollonius Type in $C^{*}$-Algebras, Abstract and Applied Analysis, 2008, doi:10.1155/2008/672618.

21. A. Najati and G. Z. Eskandani, Stability of a mixed additive and cubic functional equation in quasi-Banach spaces, J. Math. Anal. Appl., 342 (2008), 1318-1331.

22. C. Park and F. Moradlou, Stability of Homomorphisms and Derivations in $\mathrm{C}^{*}$-ternary Rings, Taiwanese J. Math., to appear.

23. Th. M. Rassias, On the stability of the linear mapping in Banach spaces, Proc. Amer. Math. Soc., 72 (1978), 297-300.

24. F. Skof, Local properties and approximations of operators, Rend. Sem. Mat. Fis. Milano, 53 (1983), 113-129. 
25. S. M. Ulam, A Collection of the Mathematical Problems, Interscience Publ., New York, 1960.

G. Zamani Eskandani, Hamid Vaezi and Y. N. Dehghan Department of Mathematics,

Faculty of Sciences,

University of Tabriz,

Tabriz, Iran

E-mail: zamani@tabrizu.ac.ir

hvaezi@tabrizu.ac.ir

nejad@tabrizu.ac.ir 